\title{
The thermal behaviour of a special heat exchanger filled with the phase change material dedicated for low - temperature storage applications
}

\author{
Agnieszka Ochman ${ }^{1, *}$, Sławomir Pietrowicz ${ }^{1}$ \\ ${ }^{1}$ Department of Thermodynamics, Theory of Machines and Thermal Systems, Faculty of Mechanical \\ and Power Engineering, Wroclaw University of Science and Technology, Wybrzeże Wyspiańskiego \\ 27 Street, 50-370 Wroclaw, Poland
}

\begin{abstract}
The work deals with an experimental and theoretical analysis of a special heat exchanger filled with the PCM (Phase Change Material) mixture of paraffin wax capsuled in the melamine-formaldehyde membrane and water, dedicated to low-temperature storage applications. In the first part of the article, the PCM topic was introduced. The next part consists information about the designed and manufactured special heat exchanger with the PCM. During the experiment as a PCM is utilized mixture of paraffin wax in microcapsules within the water. The experimental results of temperature distributions and stored energy in the heat exchanger were compared with the theoretical model proposed in the literature. In the discussion section, the authors describe a new idea of the PCM storage tank application, which could be applied e.g. in nuclear facilities.
\end{abstract}

\section{Introduction}

Generally, the thermal energy can be stored in three ways: by changing the temperature of the storage material (sensible heat storage), by changing a phase of the material (latent heat storage) or by using chemical processes. Sensible heat storage is a well-known method utilized, for example, in hot water heat storage tanks. The best-known methods of using latent heat storage are phase change materials storages. A possible solution to use chemical processes in storing energy is the utilization of oxidation and reduction of chemicals with oxygen.

A PCM is an abbreviation of Phase Change Materials. The PCM melts or crystallizes at a specified temperature. In contrast to other energy storage solutions, the PCM allows for the storage of large amounts of the thermal energy without significant temperature changes, because of high latent heat. One of the oldest and most popular PCM is, for instance, water. Low melting temperature $\left(0^{\circ} \mathrm{C}\right)$ and high value of the latent heat $(330 \mathrm{~kJ} / \mathrm{kg})$ allows for the utilization of ice in cooling systems in medicine, transport or households. The disadvantages of PCM are low thermal conductivity and sub-cooling. Generally, two main types of PCM can be used in practice: organic and inorganic. The improvement of the PCM's thermal conductivity can be achieved by creating a mixture of materials using substances

\footnotetext{
* Corresponding author: agnieszka.ochman@pwr.edu.pl
} 
with a high thermal conductivity. The most popular solution is an encapsulation of PCM and mixed it with water, which prevents stratification of mixture components during phase change. In addition, encapsulation reduces PCM chemical instability and corrosiveness. Likewise, phase change processes can be enhanced by the expansion of the heat transfer area.

One of the applications using PCM can be found in nuclear facilities where heating or cooling processes require heat storage or discharge of a large amount of excess heat. Currently, the application of PCM in nuclear facilities is undergoing intensive development. Alameri and King [4] analysed the utilization of the PCM storage as a primary coolant of the reactor. The conclusion from these analyses is that the application of PCM allows them to maintain a constant thermal power level of the nuclear reactor. Jeong and Bang [5] proposed the use of the PCM tank in a hybrid heat pipe system. A hybrid heat pipe removes decay heat from a spent nuclear fuel (SNF). The PCM can be used as a heat transfer medium between waste heat from the spent nuclear fuel and e.g. a heating network. Han et al. $[6,7]$ presented a mobile robot working during a nuclear accident. The robot is protected against irradiation by a metal cover, which caused overheating of its electronic components. The application of PCM reduced overheating and increased the robot working time from $5 \mathrm{~min}$ to $78 \mathrm{~min}$. The literature review demonstrated also the necessity of additional analyses of PCM properties and the search of new design of the PCM storage tanks for heat energy management.

This article presents unique PCM storage device construction, which can be used as a storage tank, safety heat buffer or heat exchanger. The authors performed the experimental tests to examine temperature evolution during charging and discharging processes and compared the results with the analytical solutions.

\section{Experimental setup and results}

\subsection{PCM storage tank construction}

The major problem in the PCM tanks is weak heat transfer caused by low thermal conductivity. To eliminate this problem, the special construction of PCM tank was designed, manufactured and commissioned. In the investigated device, the distance between the heat sources (pipes) and PCM layers was reduced. The idea of the PCM storage tank is shown in Fig. 1.

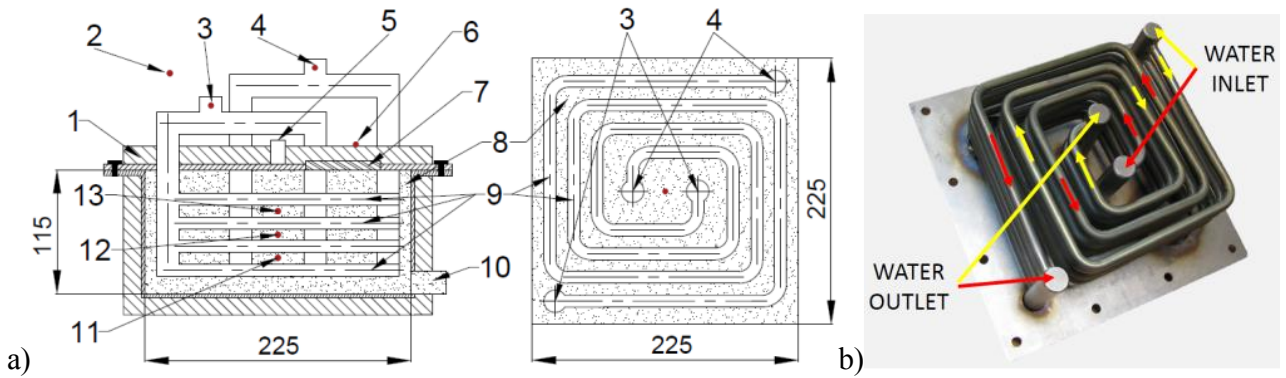

Fig. 1. a) PCM storage tank construction. 1. Insulation 2. Thermocouple measuring ambient temperature 3. Inlet collectors with $T$ inlet thermocouple 4. Outlet collectors with $T$ _outlet thermocouple 5. Cable grommet 6 . Thermocouple measuring wall temperature 7. PCM charging valve 8. PCM 9. Stainless steel tubes $\varnothing 10 \mathrm{~mm} 10$. PCM discharging valve $11 . T_{-} \mathrm{c} 3$ thermocouple $12 . T_{-} \mathrm{c} 2$ thermocouple 13 . T_c1 thermocouple; b) The configuration of the water flow

The tank is equipped with PCM charging (7) and discharging (10) valves which give the opportunity to utilize different types of PCM. Two independent tube systems can be utilized for charging and discharging stored energy. One system contains four parallel tubes connected by the inlet and outlet collectors. Both tubes systems have the same dimensions equal 
to $\varnothing 10 \mathrm{~mm}$. Small distance between tubes $(10 \mathrm{~mm})$ increase thermal conductivity between water and PCM and causes the PCM to melt congruently. In addition, the proposed design of the exchanger allows for the reduction of thermal stresses resulting from variable thermal work conditions. The tank construction allows work in different flow configurations of the working medium for charging and discharging. The article presents the thermal behaviour of the device, when both systems work in a counter flow charging or discharging, showed in Fig. $1 b$.

\subsection{Results and discussion}

To check the possibility of PCM storage tank application, the melting and crystallisation processes of PCM were investigated experimentally and theoretically. The total amount of $3.322 \mathrm{~kg}$ mixture of paraffin wax in the melamine-formaldehyde membrane (microcapsules) and water was utilized during the experiment. The properties of the PCM are: heat storage capacity of slurry: $60-72 \mathrm{~kJ} / \mathrm{kg}$, heat storage capacity of dried microcapsules: $180-192 \mathrm{~kJ} / \mathrm{kg}$, density: $900-970 \mathrm{~g} / \mathrm{L}$. The hot or cooled water flows in the tubes with an average volume flow rate equal to $6.9 \mathrm{~L} / \mathrm{min}$. The volume flow rate of the water was measured by an ultrasonic flow meter (measuring deviation: $\pm 1 \%$ ), all temperatures by $\mathrm{T}$ thermocouples with a measuring deviation: $\pm 0.5^{\circ} \mathrm{C}$. For estimation of the heat loss from the system to the environment, additional thermocouples were placed on the tank wall and outside, $10 \mathrm{~mm}$ from wall. The example of the PCM and water temperature evolutions during the heating and cooling processes are presented in Fig. 2 and Fig. 3. Green and blue dashed lines show the changes of the water temperature at the inlet and outlet of the tank ( $T$ _inlet, T_outlet). The temperature in the PCM is measured and recorded by three thermocouples called $\bar{T}_{-} c 1, T_{-} c 2$ and T_c3 (see Fig. 1a).

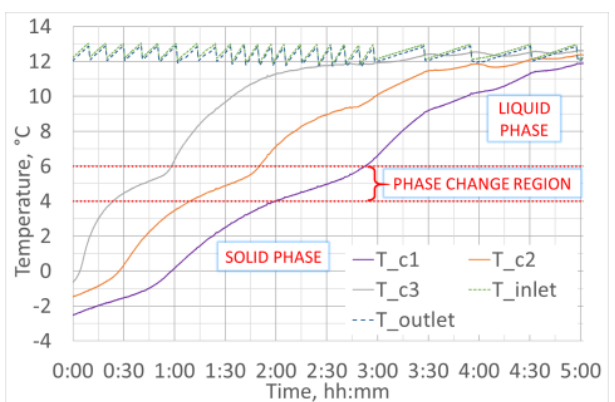

Fig. 2. The evolution of the water and PCM temperatures during the heating process. For the thermocouples (T_c1, T_c2, T_c3, T_inlet, T_outlet) localization, see Fig. 1a.

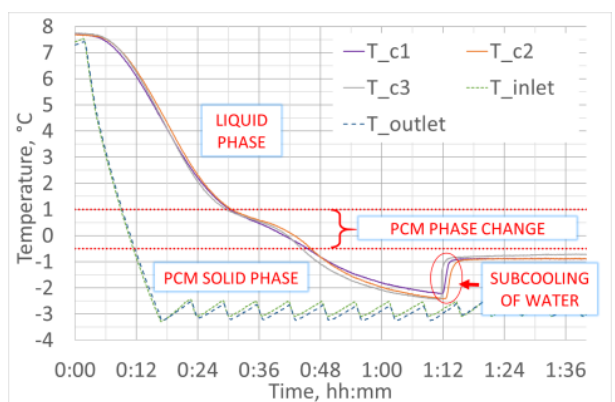

Fig. 3. The evolution of the water and PCM temperatures during the cooling process. For the thermocouples (T_c1, T_c2, T_c3, T_inlet, T_outlet) localization, see Fig. 1a.

It can be observed that the melting process starts at $4^{\circ} \mathrm{C}$ and finishes at $6^{\circ} \mathrm{C}$ and that the crystallisation process begins at $1{ }^{\circ} \mathrm{C}$ and ends at $-0,5^{\circ} \mathrm{C}$. The differences between those temperature values is called the phase change region. This region characterizes a lower temperature increase (or decrease) in time, in comparison to the temperature evolution for onephase substances. Melting process of analysed PCM is less uniform than crystallisation process. An half hour delay in the temperature distribution can be caused by an occurrence of ice fractions in the PCM material, lower thermal conductivity for dried microcapsules, thermocouple distance from the tank construction or environmental impact.

The amount of heat transfer to the PCM is presented in Fig. 4 and Fig. 5. Observed temporary power drops from the average value can be identified by the chiller hysteresis sets at $1{ }^{\circ} \mathrm{C}$. For the heating process, four peaks were observed, caused by changes in temperature stabilization next to the chiller. 


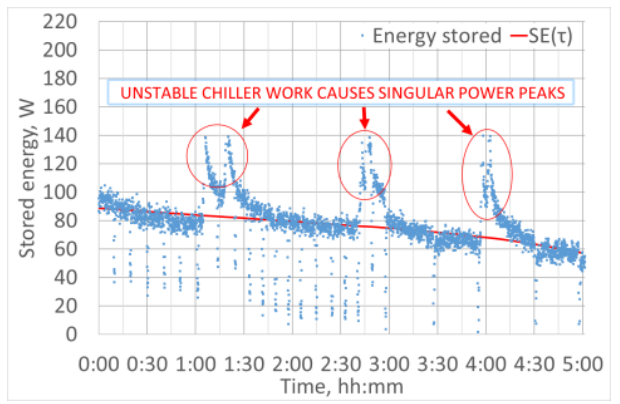

Fig. 4. The experimental results of the stored energy power during heating process (blue points) and the used fitting $S E(\tau)$ function (red solid line).

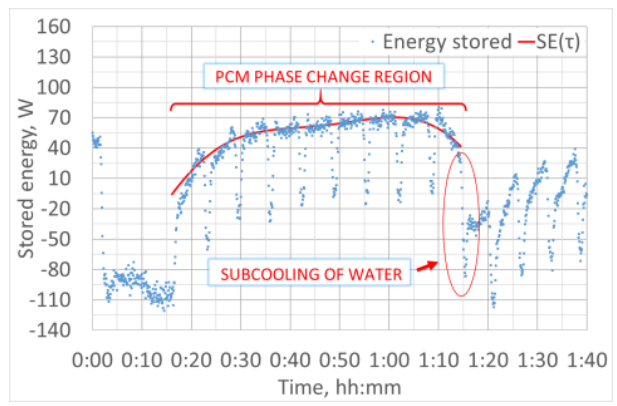

Fig. 5. The experimental results of the stored energy power during cooling process (blue points) and used the fitting $S E(\tau)$ function (red solid line).

The energy stored by the PCM is calculated on the basis of water flow balance analysis and ambient parameters. Theoretical calculations of energy stored include energy stored in the sensible heat of liquid and solid phases and heat of phase change. Due to the PCM temperature reallocation in time, the total energy stored was calculated for 5 hours of PCM heating. The results were adjusted by the approximation function 1, which is the Sum of Sine equation. Function integration over the time interval gives heat accumulated by the tank.

$$
S E(\tau)=a 1 \cdot \sin (b 1 \cdot \tau+c 1)+a 2 \cdot \sin (b 2 \cdot \tau+c 2)
$$

where: SE is a stored energy fitting function over the normalized time $\tau, \mathbf{a} 1$ and $\mathbf{a} 2$ are amplitude parameters, b1, b2 - frequency parameters, c1, c2 - phase constant parameters for each sine wave.

The experimental results give $1334 \mathrm{~kJ}$ in comparison with a theoretical model where stored heat is about $1422 \mathrm{~kJ}$. The relative error between experiment and theoretical results is equal to $6.2 \%$. Theoretical model assumes that melting of dispersed paraffin wax into the microcapsule requires about $598 \mathrm{~kJ}$ of energy, melting of ice $664 \mathrm{~kJ}$ energy and sensible heat of the mixture of dispersed paraffin wax within the water is about $160 \mathrm{~kJ}$.

Cooling of the PCM is more uniform. The process of endothermic crystallisation allows calculate power only for phase change region. The results for PCM crystallization were approximated by Sum of Sine function 1 and integrated over the time interval. For theoretical analysis, the value of PCM slurry crystallisation heat transfer is about $199 \mathrm{~kJ}$. Research results for endothermic process give $189 \mathrm{~kJ}$. In this case, the relative error is $5.0 \%$.

\section{PCM storage tank application}

The PCM storage tanks are usually utilized in solar power plants as a thermal energy storage for night-time demand $[8,9]$. In addition, the PCMs are commonly applied in spacecraft thermal control systems for low and high temperature applications [10].

The storage tank filled with microcapsules of PCM can be utilized in low temperature systems, with low power demand, like laboratory equipment for cooling, heating or stabilizing temperature processes. One of the ideas of the low temperature PCM storage application is cooling the pulsating heat pipe (PHP) systems, which require stable thermal parameters [11]. Application of movable cover and stainless steel construction allows conduct research for different PCM types and mixtures of PCM, also with components increasing their conductivity.

PCM thermal system can be used for maintaining a stable temperature in the nuclear facilities. Most of the nuclear systems work at a higher temperature range than presented in the paper. However, the described idea can also be used for this purpose, for example, by replacing paraffin wax with a high temperature PCM such as salts. Resizing the tank permits 
an increase the amount of utilizable PCM in order to achieve a higher heat storage capacity. The system allows storage of excess energy during low electricity demand and can use it during peak consumption time. This solution enhances safety and reactor efficiency by maintaining a stable level of the reactor thermal power generation.

Moreover, the PCM tank can be a part of the natural convection passive cooling system belonging to the reactor safety cooling system [12]. To increase the temperature difference between the reactor and the cooler, and therefore increase the efficiency of the natural convection, a PCM storage tank can be utilized as a cooler of the circulating fluid. The high latent heat of the PCM allows for the storage of a large amount of energy.

\section{Conclusions}

In this work, the thermal behaviour of heating and cooling processes in a special heat exchanger filled with the PCM was studied.

For the analysed PCM it was observed differences in the melting and crystallization temperatures and phase change energy demand. The crystallization process has almost uniform temperature distribution (within the range of $-0.5^{\circ} \mathrm{C} \div 1^{\circ} \mathrm{C}$ ) due to the mixture of paraffin wax with water and applied low distances between the tank tubes in the heat exchanger construction. It was observed that the endothermic process of crystallization causes that utilized PCM needs lower energy for discharging (about $200 \mathrm{~kJ}$ ) than during charging (about $600 \mathrm{~kJ}$ ). This is an advantage for application where the PCM storage tank should cool down the circulating fluid in the system and where the PCM storage tank will be utilized for temperature stabilisation.

Additionally in the article, the potential applications of the PCM storage tank in nuclear facilities is proposed. PCM in nuclear facilities can be utilized in different fields to store excess energy for later usage in the latent heat storage, to stabilize the temperature of different processes or to avoid the overheating of electronic devices.

\section{References}

1. H. Mehling, L. F. Cabeza, Heat and cold storage with PCM (Springer - Verlag Berlin Heidelberg, 2008)

2. J. Gomez, G. C., High - Temperature Phase Change Materials (PCM) Candidates for Thermal Energy Storage (TES) Applications (National Renewable Energy Laboratory, September 2011)

3. M. M. Kenisarin, Renewable and Sustainable Energy Reviews, 14, 955 - 970 (2010)

4. S. A. Alameri, J. C. King, 2013 International Nuclear Atlantic Conference - INAC 2013 (Recife, PE, Brazil, November 24-29, 2013)

5. Y. S. Jeong, I. C. Bang, Appl. Therm. Eng., 96, 277-285 (2016)

6. Y. Han, W. Luan, Y. Jiang, X. Zhang, The 7th International Conference on Applied Energy - ICAE2015, Energy Procedia, 75, 3301 - 3306 (2015)

7. Y. Han, W. Luan, Y. Jiang, X. Zhang, Appl. Therm. Eng., 101, 224-230 (2016)

8. A. Agarwal, R. M. Sarviya, 5th International Conference of Materials Processing and Characterization (ICMPC 2016), 4, 779 - 789 (2017)

9. A. Sharma, V.V. Tyagi, C.R. Chen, and D. Buddhi, Renewable and Sustainable Energy Reviews, 13, 318 - 345 (2009)

10. T. D. Swanson, G. C. Birur, Appl. Therm. Eng., 23, 1055-1065 (2003)

11. C. Czajkowski, S. Pietrowicz, A. Nowak, Współ czesne problem termodynamiki, Wydawnictwo Instytutu Techniki Cieplnej, 651 - 665 (2017)

12. H. Abdillah, G. Saputra, Novitrian, S. Permana, J. Phys.: Conf. Ser., 877 (2017) 